\title{
Downregulation of CCL2 induced by the upregulation of microRNA-206 is associated with the severity of HEV71 encephalitis
}

\author{
GUANGYOU ZHANG ${ }^{1,2}$, JIWEN WANG $^{2,3}$, GUO YAO $^{1}$ and BAOHAI SHI ${ }^{1}$ \\ ${ }^{1}$ Department of Pediatrics, Taian Central Hospital, Taian, Shandong 271000; ${ }^{2}$ Department of Pediatrics, \\ Qilu Hospital of Shandong University, Jinan, Shandong 250012; ${ }^{3}$ Department of Neurology, Shanghai \\ Children's Medical Center, Jiaotong University School of Medicine, Shanghai 200127, P.R. China
}

Received March 8, 2016; Accepted February 20, 2017

DOI: $10.3892 / \mathrm{mmr} .2017 .7142$

\begin{abstract}
MicroRNAs (miRNAs) have been investigated widely as key regulators of gene expression in different diseases by affecting the miRNA-mediated regulatory function. Human enterovirus 71 (HEV71) can cause a series of human diseases, including encephalitis. Chemokine (C-C motif) ligand 2 (CCL2) is one of the important genes involved in regulating inflammation. However, the mechanisms underlying HEV71 encephalitis mediated by CCL 2 remain to be elucidated. In the present study, reverse transcription-quantitative polymerase chain reaction analysis was used to determine the expression level of miR-206 and the mRNA expression of CCL2 in samples. Western blot analysis was used to detect the protein levels of CCL2. A luciferase assay was used to verify the miR-206 target site in CCL2. A CCK-8 assay and flow cytometry were used to determine cell proliferation and apoptosis. The results demonstrated that miR-206 was downregulated in severe HEV71 encephalitis. Using bioinformatics analysis, miR-206 was predicted to target the human CCL2 3'-untranslated region (3'-UTR). A dual-luciferase assay demonstrated that miR-206 downregulated the expression of CCL2 by directly targeting its 3'-UTR, whereas CCL2 3'-UTR mutations completely eliminated its interaction with miR-206. The expression levels of miR-206 and CCL2 were inversely correlated in cerebrospinal fluid. The expression of exogenous miRNA, which mimicked miR-206 miRNA, decreased the protein and mRNA levels of CCL2, whereas the suppression of endogenous miR-206 resulted in an increase of the protein and mRNA levels of CCL2. The present study also found that miR-206 promoted NPC cell proliferation and reduced the apoptosis of NPC cells via CCL2. The mechanism
\end{abstract}

Correspondence to: Dr Jiwen Wang, Department of Pediatrics, Qilu Hospital of Shandong University, 107 West Wenhua Road, Jinan, Shandong 250012, P.R. China

E-mail: encephalitis1@163.com

Key words: C-C motif chemokine ligand 2, microRNA-206, severity, HEV71, encephalitis is likely to involve suppression of the expression of miR-206 and upregulation of the expression of CCL2, important in regulating the progress of HEV71 encephalitis. In conclusion, miR-206 may be useful in the prognosis and treatment of HEV71 encephalitis.

\section{Introduction}

As a single-stranded, positive-sense RNA virus, human enterovirus 71 (HEV71) belongs to the genus Enterovirus, family Picornaviridae (1). HEV71 contributes to neurological complications, meningitis, encephalitis, and contributes to mortality rates in young children and infants; it may be one of the pioneer pathogens of hand-foot-and-mouth disease (HFMD), which is a life-threatening, newly emerging pathogen in children, specifically in the Asia-Pacific region (2).

A wide spectrum of clinical manifestations can be induced by HEV71 infection, including respiratory infections, mild febrile illness, characteristic cutaneous diseases, including HFMD, herpangina, and severe complications, including acute flaccid paralysis, brainstem encephalitis, encephalitis, hepatitis and myocarditis (3). A systemic inflammatory response syndrome, which is produced by the release of cytokines and chemokines, and/or direct lesions, can result in severe complications in HEV71 (3). The overexpression of chemokine cascades, including interleukin (IL) 8, chemokine (C-C motif) ligand 2 (CCL2), and C-X-C motif chemokine 10, in the central nervous system (CNS) compartment are reported to have a significant effect on elicitation of the immune response to HEV71 (4).

Chemokines are important secondary inflammatory mediators released in response to stimuli. As second-order cytokines, they have specialized functions in inflammation. The role of a number of these specialized mediators in the human intervertebral disc remain to be elucidated. Investigations by Schroeder et al on disc degeneration with acute or chronic pain focused on chemokine profiles (5). It was reported that small inducible cytokine A2 and CCL2, also known as monocyte chemotactic protein-1, may be associated with inflammatory mediators in low back pain and the resorption of herniated disc tissue. Studies by Kikuchi et al and Yoshida et al found, through laboratory experiments, that 
treatment of human annulus cells with $1,25(\mathrm{OH})_{2} \mathrm{D}_{3}$, led to significant decreases $(\mathrm{P}=0.002)$ in the production of vascular endothelial growth factor and CCL2 (6-8).

Non-coding, single-stranded RNA nucleotides with a length of 18-25 are found in the genomes of all multicellular organisms and certain viruses, and microRNAs (miRNAs) are a class of these nucleotides (9). miRNAs bind mRNAs to complementary sequences in the mRNA to functionally result in the degradation of mRNAs or prevention of the translation of mRNAs (10). Therefore, miRNAs can regulate immunity, oncogenesis, apoptosis, development, proliferation, cell differentiation and cell cycle (11-13).

The differential expression of miRNAs, including miR-206, have been identified in severe HEV71 encephalitis (14), and it has been shown that levels of CCL2 are associated with the severity of HEV71 encephalitis $(15,16)$. In the present study, by performing an online miRNA database search (www.mirdb .org), CCL2 was identified as a virtual target of miR-206. The present study aimed to validate CCL2 as a target of miR-206, and to investigate whether miR-206/CCL2 are functionally involved in determining the severity of HEV71 encephalitis.

\section{Materials and methods}

Subjects. The present study recruited 74 suspected cases of HEV71 encephalitis, including mild cases $(n=40)$ and severe cases $(n=34)$, at the Department of Pediatrics, Qilu Hospital of Shandong University (Shandong, China). All patients were admitted between August 2013 and December 2014 (54 males and 20 females; age, 15.2-41.2 months old; weight, 10.39-15.67 kg). All 74 cases were diagnosed as HEV71 encephalitis. The patients were divided into two groups, comprising a HEV71-infected encephalitis group and an HFMD without complications group. HFMDs were diagnosed according to the following clinical features: Typical ulcers on lips, and rashes on the hands and feet. Reverse transcription-quantitative polymerase chain reaction (RT-qPCR) analysis was performed to detect the HEV71 virus infection. On the day of admission, throat swabs or cerebrospinal fluid (CSF) samples were collected from each subject for RNA extraction. A diagnosis of encephalitis was made when subjects met the following criteria: White blood cell count $>5 / \mathrm{mm}^{3}$ in the CSF; parenchymal damage detected by MRI or CT scan. Each subject went through a regular physical examination and other laboratory tests in the following 3 days, from which baseline values of clinical and laboratory characteristics were collected. The study protocol was approved by the Clinical Research Ethics Committee of Shandong University. Written Informed consent was obtained from each subjects' guardian prior to commencement of the study.

CCL2 ELISAs. CSF (2 ml) was collected and centrifuged at $1,600 \mathrm{x} \mathrm{g}$ for $10 \mathrm{~min}$ to remove particles. The levels of CCL2 were determined using a Cusabio ELISA kit (Cusabio Biotech Co., Ltd., Wuhan, China) according to the manufacturer's protocol. In brief, the kit contained a microplate precoated with monoclonal antibody against CCL2 and a peroxidase-bonded anti-CCL2 polyclonal antibody. The detecting sensitivity was $5 \mathrm{pg} / \mathrm{ml}$.
$R N A$ isolation and $R T-q P C R$ analysis. Total RNA were extracted using an RNA extraction kit (Ambion; Thermo Fisher Scientific, Inc., Waltham, MA, USA). The RNA extract was used to generate the first strand cDNA using the SuperRT cDNA Synthesis kit (Kangwei Biotech Co., Ltd., Beijing, China). The qPCR reaction (total volume, $20 \mu \mathrm{l}$ ) was performed using UltraSYBR Green qPCR Master mix (with ROX I) (Kangwei Biotech Co., Ltd.) and the LightCycler 480 Real-Time PCR system (Roche Diagnostics $\mathrm{GmbH}$, Mannheim, Germany). The primer sequences were as follows: CCL2 (forward 5'-ATGCAGTTAATGCCCCACTC-3', reverse 5'-TTCCTTATTGGGGTCAGCAC-3'), miR-206 (3'-UGG AAUGUA AGGAAGUGUGUGG-5'), GAPDH (forward 5'-GAAGATGGTGATGGGATTTC-3', reverse 5'-GAAGGT GAAGGTCGGAGTC-3'), U6 (forward 5'-CTCGCTTCG GCAGCACATATACT-3', reverse 5'-ACGCTTCACGAATTT GCGTGTC-3'). All samples were run in duplicate and the reaction conditions were as follows: pre-denaturation at $94^{\circ} \mathrm{C}$ for $5 \mathrm{~min}$, followed by 40 cycles of denaturation at $94^{\circ} \mathrm{C}$ for $30 \mathrm{sec}$, annealing at $50^{\circ} \mathrm{C}$ for $40 \mathrm{sec}$ and extension at $72^{\circ} \mathrm{C}$ for $40 \mathrm{sec}$. The $2^{-\Delta \Delta \mathrm{Cq}}$ relative quantification method was used to calculate the expression levels of miRNA (17). The expression of U6 was used as an internal control. Values were expressed as fold differences of the expression of miR-206 and CCL2 relative to that of U6.

Cell culture and transfection. U-251 cells (Ybiotech, Beijing, China) were maintained in DMEM (HyClone; GE Healthcare Life Sciences, Logan, UT, USA) supplemented with $10 \%$ fetal bovine serum (Gibco; Thermo Fisher Scientific, Inc.) at $37^{\circ} \mathrm{C}$ in $5 \% \mathrm{CO}_{2}$ humidified atmosphere. miR-206 mimics or inhibitor and CCL2 small interfering (si)RNA (50 nM) (Shanghai GenePharma Co., Ltd., Shanghai, China) were transfected into the U-251 cells ( $1 \times 10^{5}$ cells) using Lipofectamine 2000 transfection reagent (Thermo Fisher Scientific, Inc.) in accordance with the manufacturer's protocol. In addition, scrambled controls were transfected into cells as negative controls. The transfection efficiency of the U-251 cells was confirmed using RT-qPCR analysis.

Vector construction and mutagenesis. The 3'untranslated region (UTR) of CCL2 containing the putative binding site of miR-206 was identified by searching the online miRNA database $(18,19)$. The CCL2 3'UTR was amplified using PCR, and the PCR products were isolated using agarose gel (2\%). The fragments were then purified and inserted into a pGL3-control vector (Promega Coproration, Madison, WI, USA). Mutagenesis was performed for the same site and inserted into the control vector (Promega Corporation) using PCR. The miR-206 primers were added into the reverse transcription system. The miRNA reverse transcription program is consistent with the common mRNA procedures (20).

Luciferase assay. A dual-luciferase reporter assay (Promega Corporation) was used to investigate the effects of the miR-206 (targeting the putative 3'UTR binding site) on luciferase activity. In brief, the cells were seeded into a 96-well plate $\left(3 \times 10^{4}\right.$ cells $/ \mathrm{ml} ; 200 \mu \mathrm{l} /$ well) overnight prior to transfection. On the day of transfection, the U-251 cells were cotransfected with the wild-type (WT) or mutant type (MT) 
vector and miR-206 or the negative control vector. At 2 days post-transfection, the cells were subjected to dual-luciferase reporter assays. The luciferase activity of a Renilla control plasmid was also analyzed as in internal control. The results were recorded as ratios of miR-206 luciferase activity relative to that of Renilla.

Western blot analysis. Western blot analysis was performed to assess the effect of miR-206 on protein expression in the U-251 cells. The U-251 cells were lysed and purified, and the protein level was determined using an Enhanced BCA Protein Assay kit (Sigma-Aldrich; Merck Millipore, Darmstadt, Germany). The extracts, containing $25 \mu \mathrm{g}$ of total proteins, were loaded on $10 \%$ SDS-polyacrylamide gel. Following electrophoresis, the protein blots were transferred onto a polyvinylidene difluoride (PVDF) membrane. The membrane was washed with PBS and then blocked in $10 \%$ skimmed milk at $4^{\circ} \mathrm{C}$ overnight. The PVDF membranes were incubated with the following antibodies at $4^{\circ} \mathrm{C}$ overnight: Rabbit anti-CCL2 (IC479A; 1:700; R\&D Systems, Inc., Minneapolis, MN, USA) or rabbit anti $\beta$-actin (a8227; 1:300; Abcam, Cambridge, UK), and subsequently incubated with goat anti-rabbit IgG (CW0111S; 1:2,200; Kangwei Biotech Co., Ltd.) for $2.5 \mathrm{~h}$ at room temperature. The conjugated proteins were visualized using an enhanced chemiluminescence detection kit (Pierce; Thermo Fisher Scientific, Inc.). Fluor Chen 2.0 software (Olympus, Yokohama, Japan) was used to analyzed the density values. $\beta$-actin was used as an internal control, with values presented as the expression of CCL 2 relative to that of $\beta$-actin.

Statistical analysis. For each experiment, replicate samples were subjected to analyses at least three times and the results were compared using Student's t test. All data analyses were performed using SPSS 19.0 (IBM SPSS, Armonk, NY, USA). Comparisons among more than three groups were performed using one-way, two-way or three-way analysis of variance (ANOVA) without repeated measures, as appropriate. Comparisons among less than three were performed using one-way ANOVA followed by Dunnett's post hoc test. Comparisons among three or groups were performed using two- or three-way ANOVA followed by a stratified independent t-test with Bonferroni corrections for multiple comparisons. $\mathrm{P} \leq 0.05$ was considered to indicate a statistically significant difference. The values from all experiments are expressed as the mean \pm standard deviation.

\section{Results}

miR-206 is downregulateed in severe HEV71 encephalitis. To identify miRNAs involved in regulating the severity of HEV71 encephalitis, the present study compared miRNA expression levels between severe HEV71 encephalitis and mild HEV71 encephalitis samples. It was found that miR-206 was consistently downregulated in severe HEV71 encephalitis (Fig. 1). Therefore, the present study focused on miR-206, and it was hypothesized that miR-206 may regulate the transformation of mild HEV71 encephalitis into severe HEV71 encephalitis.

CCL2 expression transcripts are targeted by miR-206. One of the primary challenges in examining miRNAs is to identify

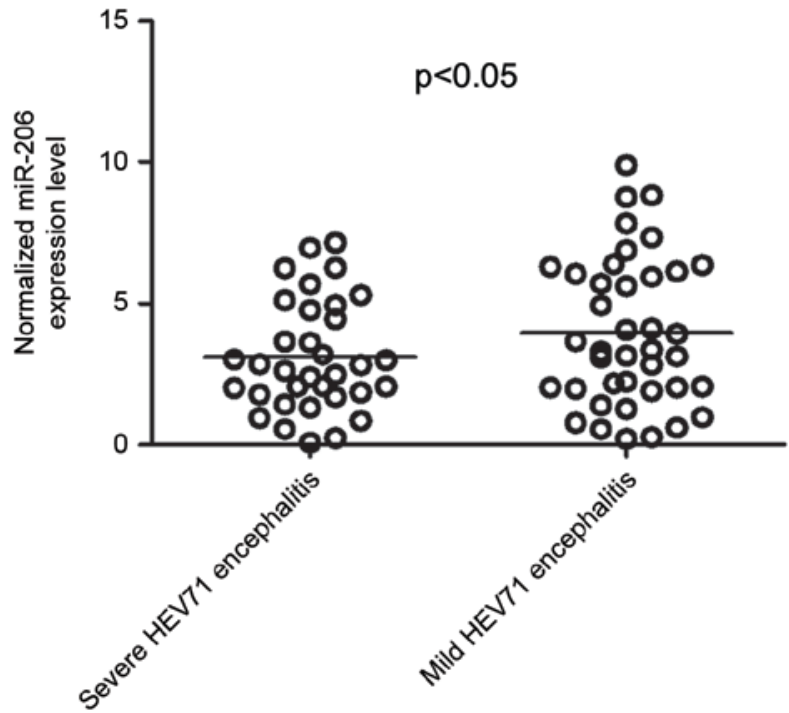

Figure 1. miR-206 is downregulated in severe HEV71 encephalitis. Expression of miR-206 in RNA samples extracted from HEV71 encephalitis tissue with severe $(n=34)$ or mild $(n=40)$ symptoms. Expression of miR-206 was normalized to that of U6. miR, microRNA.

\begin{tabular}{lc} 
hsa-miR-206 & \multicolumn{3}{c}{ 3'-UGGAAUGUAAGGAAGUGUGUGG-5' } \\
Wt-CCL2 & 5'-ACCT TAC AATT TTT TT TAAG AA-3' \\
hsa-miR-206 & 3'-UGGAAUGUAAGGAAGUGUGUGG-5' \\
$\vdots$ & $\vdots$ \\
Mt-CCL2 & 5'-GUUCCGUCATT TTT TT TAAG AA-3'
\end{tabular}

Figure 2. Schematic showing the predicted miR-206 binding sites in the 3 'untranslated region of CCL2 mRNA. The Mt construct was produced by eliminating the base pairing between the miRNA and its target. miR, microRNA; CCL2, (C-C motif) chemokine ligand 2; Wt, wild-type; Mt, mutant type.

target genes and correlate their downregulation with cellular properties. Computational algorithms have been used to predict putative miR-206 targets based on complementarity to the 3'UTR of the target mRNA. Using bioinformatics analysis, miR-206 was predicted to target the human CCL2 3'-UTR (Fig. 2). To investigate whether miR-206 is a regulator of CCL2, further experiments were performed. Firstly, luciferase reporter vectors were constructed containing a WT or MT CCL2 3'-UTR downstream of Renilla luciferase (Fig. 2). The miR-206 mimic and corresponding reporter vectors were co-transfected into U-251 cells, and a dual-luciferase assay was performed $48 \mathrm{~h}$ following transfection. The results of the dual-luciferase assay showed that miR-206 significantly suppressed the expression of luciferase if the reporter vector contained the WT CCL2 3'-UTR. By contrast, target region mutation completely eradicated this interaction (Fig. 3). To determine whether miR-206 disrupted the expression of CCL2 in a human cell line, miR-206 mimics were transfected into U-251 cells, with blank and scramble serving as a negative control, and CCL2 siRNA serving as a positive control. The data showed that the mRNA and protein expression levels of CCL2 were suppressed by the miR-206 mimic (Fig. 4A and B). Similar experiments were performed using an miR-206 inhibitor, and 


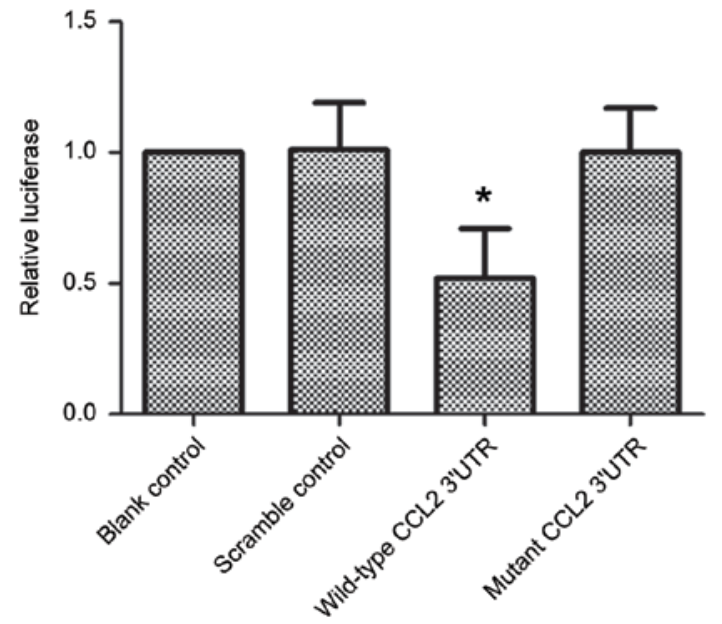

Figure 3. Reporter gene containing the predicted miR-206 binding site in its 3'UTR, with either wild-type or mutant pairing regions shows suppressed luciferase activity. U-251 cells were co-transfected with miR-206 mimic and the indicated CCL2 3'UTR-luciferase reporter. Blank and scramble served as a negative control. Luciferase activity was assayed $48 \mathrm{~h}$ later. The data are presented as the mean \pm standard deviation, normalized to the blank control, of three independent experiments. ${ }^{*} \mathrm{P}<0.05$ vs. scramble control group. CCL2, (C-C motif) chemokine ligand 2; 3'UTR, 3'untranslated region; miR, microRNA.
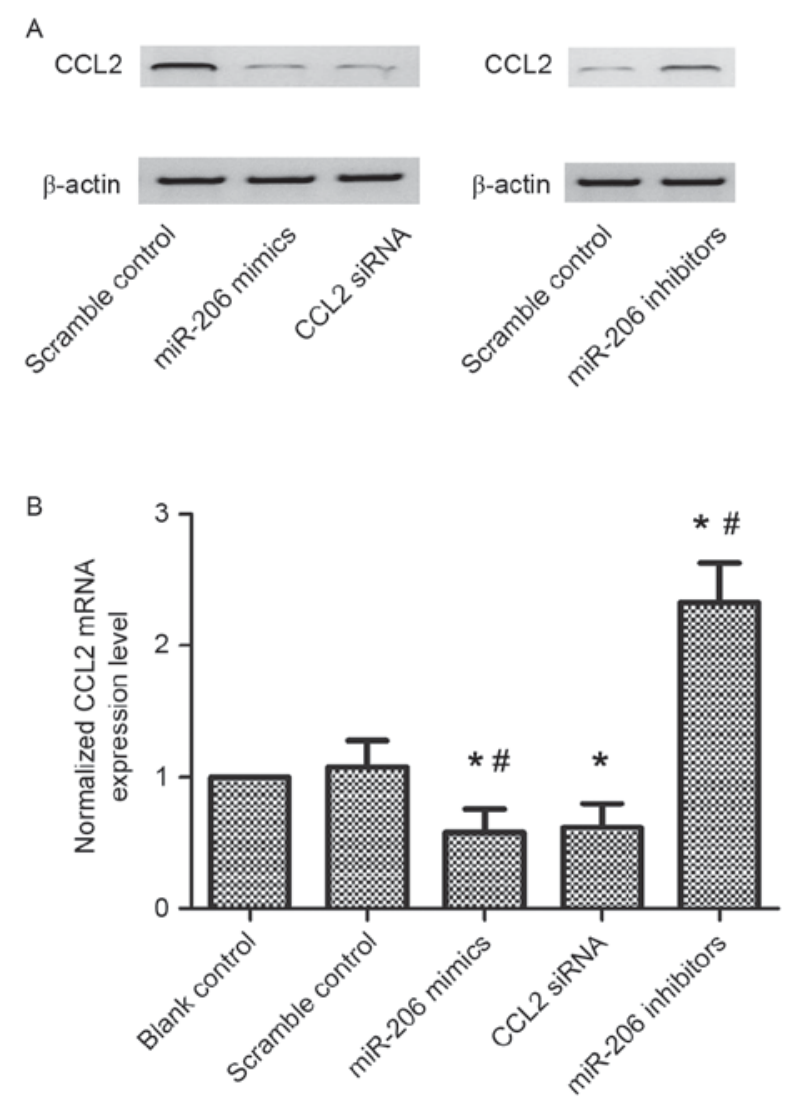

Figure 4. Protein and mRNA levels of CCL2 are inhibited by miR-206. miR-206 mimics or inhibitor were transfected into U-251 cells, respectively. (A) Western blot was used to analyze the protein levels of CCL2. (B) Relative mRNA expression levels of CCL2 were determined using reverse transcription-quantitative polymerase chain reaction analysis. Blank and scramble controls were used as a negative control. CCL2 siRNA served as a positive control. Values are presented as the mean \pm standard deviation. " $\mathrm{P}<0.05$ vs. scramble control group; ${ }^{\text {P }}<0.05$ vs. CCL2 siRNA group. CCL2, (C-C motif) chemokine ligand 2; miR, microRNA; CSF, cerebrospinal fluid; siRNA, small interfering RNA.

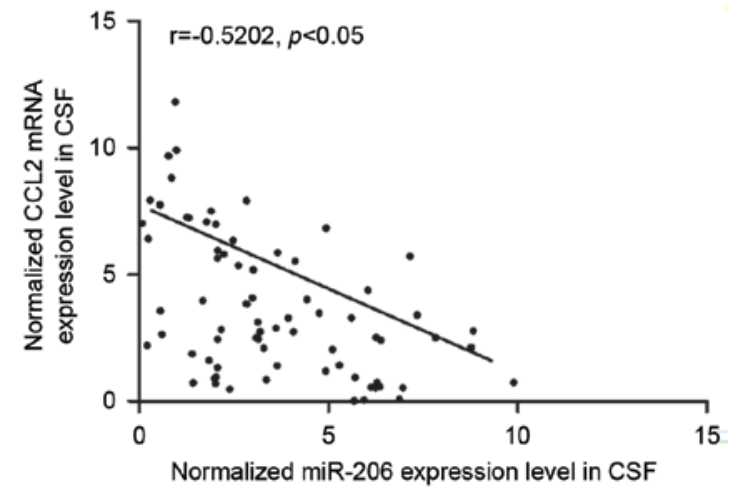

Figure 5. Expression levels of miR-206 and CCL2 are inversely correlated. The expression levels of miR-206 and CCL2 were measured in glioma samples using reverse transcription-quantitative polymerase chain reaction analysis. U6 and GAPDH served as internal normalized references for miR-206 and CCL2, respectively. The association between the expression levels of miR-206 and CCL2 were evaluated using Pearson rank correlation analysis. The results indicated a statistically significant inverse correlation $(r=-0.5202$; $\mathrm{P}<0.05$ ). CCL2, (C-C motif) chemokine ligand 2; miR, microRNA; CSF, cerebrospinal fluid.

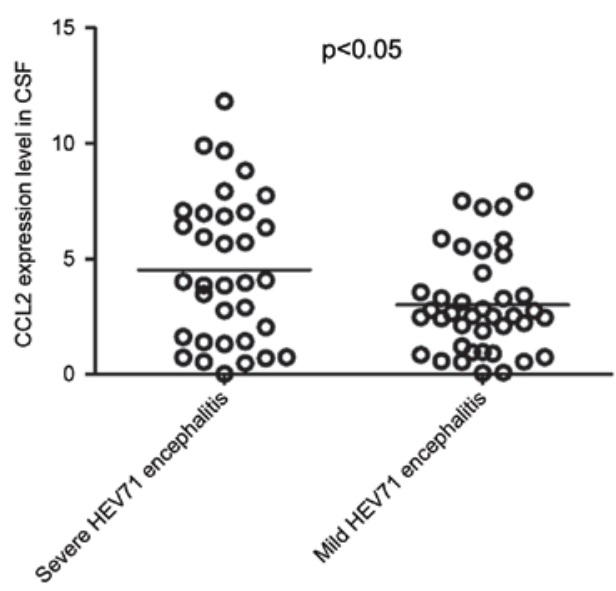

Figure 6. CCL is upregulated in CSF of patients with severe HEV71 encephalitis. Expression levels of CCL2 were determined in RNA samples extracted from CSF from patients with severe $(n=34)$ or mild $(n=40)$ HEV71 encephalitis symptoms. Expression of CCL2 was normalized to GAPDH. CCL2, (C-C motif) chemokine ligand 2; CSF, cerebrospinal fluid.

the decrease in miR-206 led to an increase in the mRNA and protein levels of CCL2 (Fig. 4A and B). To determine whether miR-206 negatively regulates endogenous CCL2 in human HEV71 encephalitis, the expression levels of miR-206 and CCL2 were measured in the CSF samples using RT-qPCR analysis. The association between the expression of miR-206 and CCL2 were also evaluated using Pearson rank correlation analysis (Fig. 5). The results indicated a statistically significant inverse correlation between miR-206 and CCL2, indicating that miR-206 suppressed the expression of CCL2 in the human brain. These results showed that miR-206 directly targeted the CCL2 3'UTR and suppressed the expression of CCL2.

Expression of CCL2 is upregulated during the transformation of mild to severe HEV71 encephalitis. The present study hypothesized that the reduction in miR-206 leads to a further increase in the expression of CCL2 in severe HEV71 

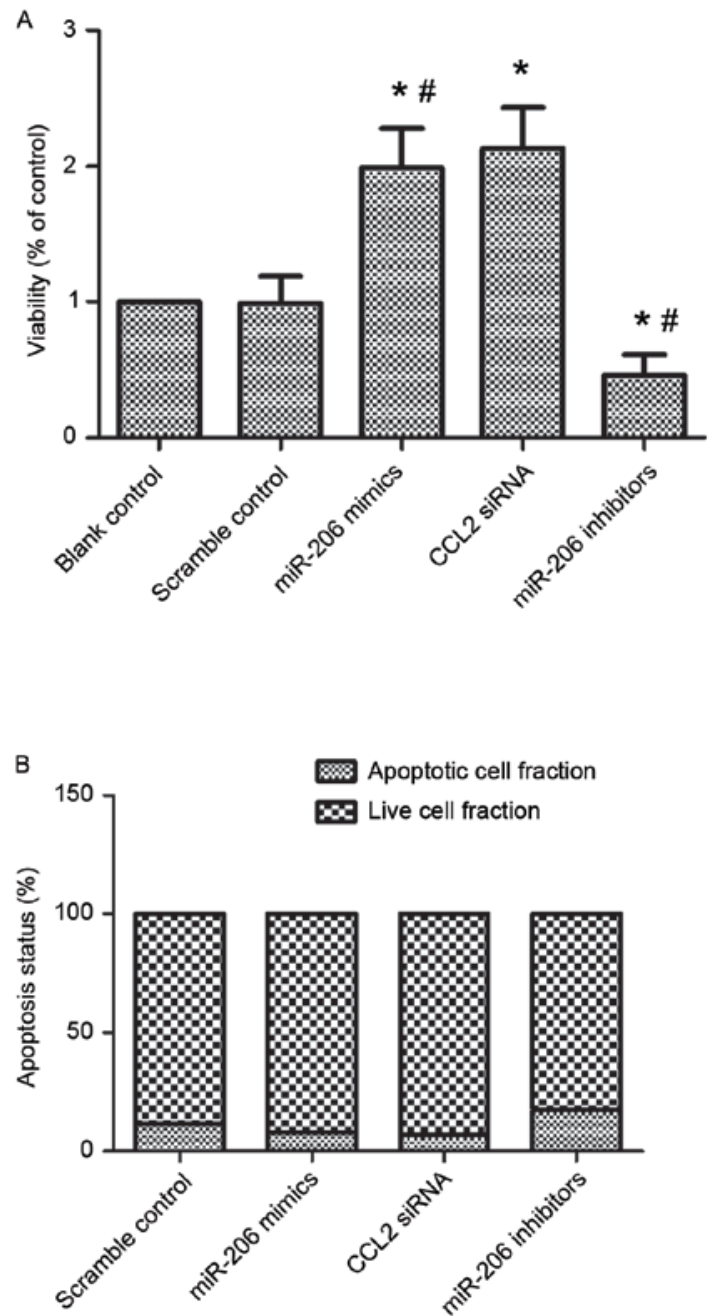

Figure 7. Effects of miR-206 on the viability and apoptosis of NPC cells. (A) miR-206 mimics or inhibitor were transfected into NPC cells. A cell counting kit- 8 assay was used to determine the effect of miR-206 on cell viability. Blank and scramble controls served as a negative control, siRNA against CCL2 served as a positive control. (B) miR-206 mimics or inhibitor were transfected into NPC cells. Apoptotic assay were performed to determine the effect of miR-206 on cell apoptosis. Scramble served as a negative control, siRNA against CCL2 served as a positive control. "P<0.05 vs. scramble control group; ${ }^{\prime} \mathrm{P}<0.05$ vs. CCL2 siRNA group. CCL2, (C-C motif) chemokine ligand 2; miR, microRNA; CSF, cerebrospinal fluid; siRNA, small interfering RNA.

encephalitis. To support this, RT-qPCR analysis was used to determine the mRNA expression level of CCL2 in the two groups (Fig. 6). The results showed that the expression of CCL2 was upregulated in the transformation of mild to severe HEV71 encephalitis.

miR-206 affects cell proliferation and apoptosis by regulating the expression of CCL2. CCL2 is important in regulating inflammation, and high expression levels of CCL2 indicate severe inflammation. Consequently, the present study hypothesized that miR-206 also regulates cell proliferation and apoptosis. To confirm this, the miR-206 mimics and inhibitor were introduced into the U-251 cells, with blank and scramble siRNA as a negative control, and CCL2 siRNA as a positive control. The results showed that miR-206 induced U-251 cell proliferation and reduced apoptosis (Fig. 7).

\section{Discussion}

The miRNA-mediated regulation of chemokines allows for an alternative classification system of chemokines and varies markedly between individual chemokines. The disturbed flow combining with oxidized low-density lipoprotein suppresses the miR-92a expression levels in a signal transducer and activator of transcription 3-dependent manner and thus increases the release of endothelial CCL2 (21). The miR-92a-mediated repression of the target suppressor of cytokine signaling (SOCS) 5 also attenuates the expression of CCL2, which is elevated by limiting endothelial inflammation myeloid-specific deficiency of KLF2 in mice, by reducing the expression of miR-150 (22), which negatively regulates miR-124a to target CCL2. CCL2 can be induced by stimulation from IL-1ß), and it increases the endothelial expression of miR-146a/b, targeting IL-1 receptor-associated kinase $1 / 2$ and tumor necrosis factor receptor associated factor 6 . If monocytes transmigrate into the intima, they differentiate into macrophages, and secrete CCL2 further propagating inflammation. In inflammatory macrophages, CCL2 is expressed by miR-155, and in macrophages of atherosclerotic lesions this expression is upregulated (23). In the present study, it was found that miR-206 was consistently downregulated in severe HEV71 encephalitis (Fig. 1). Therefore subsequent investigations focused on miR-206. It was also shown that the expression of CCL2 was upregulated during the transition between mild and severe HEV71.

As critical regulators, miRNAs contribute to inflammatory cytokine signaling, and adult skeletal muscle differentiation and maintenance (24-27). miR-206, miR-133a, miR-133b and miR-1 act as critical regulators for myoblast-to-myocyte differentiation in order to regulate the multiple genes affecting myogenesis, including serum response factor, myogenin and myogenic differentiation antigen $1(27,28)$. It has also been reported that miRNAs are critical in regulating innate and adaptive immune responses. For example, miR-221 and miR-155 target SOCS-1, following lipopolysaccharide (LPS) stimulation of cells, which regulates the production of cytokines in human dendritic cells (29). In addition, miR-101 negatively regulates the expression of MKP-1 in LPS-stimulated macrophages and thus positively regulates the production of inflammatory cytokines (30). Therefore, miR-34a, miR-98 and miR-147 are negative regulators of inflammation for LPS-induced macrophages (31). It was previously reported that miR-146a, miR-200b and miR-200c regulate the production of proinflammatory cytokines through Toll-like receptor 4 signaling (32). In the present study, the results of the dual-luciferase assay showed that miR-206 significantly suppressed luciferase expression when the reporter vector contained a WT CCL2 3'-UTR. By contrast, the MT target regions completely eradicated this interaction. In addition, a statistically significant inverse correlation was found between miR-206 and CCL2, which indicated that miR-206 suppressed the expression of CCL2 in the human brain. These results showed that miR-206 directly targeted the CCL2 3' UTR, suppressing the expression of CCL2.

Banisadr et al found that the presence of conserved adjacent cysteine residues can be used to define chemokines, including CCL2 and CCL5, which belong to the CC subfamily, and that chemokines are primarily associated with $\mathrm{T}$ cells, macrophages, 
and the recruitment of monocytes (33). In addition, Carr showed that CCL2 is produced in response to infection or injury, and is chemotactic for memory $\mathrm{T}$ cells, dendritic cells and monocytes (34). Banisadr et al and Hickman and Khoury found that resident CNS cell populations, including microglia, neurons and astrocytes, can produce CCL2, and Carrillo-de Sauvage reported that CCL 2 can mediate neuroinflammatory processes, including T-cell extravasation, into the brain $(33,35,36)$. Following infection with viruses, for example West Nile virus, herpes virus and Dengue fever virus, as a chemoattractant for monocytes and $\mathrm{T}$ cells, CCL5 is involved in neuroinflammatory responses (37-39). It was observed in the experimental infection of mice with an abortive RABV by Phares et al, that the upregulation of various inflammatory mediators, including chemokines CCL2, CCL5 and CXCL10, precedes cellular recruitment and virus clearance (40). In addition, infection with acute RABV isolates, including EBLV-2 and the pathogenic silver-haired bat virus, upregulates the levels of chemokine mRNA transcripts in mice (41-43). In the present study, miR-206 mimics were transfected into U-251 cells, with blank and scramble siRNAs as a negative control, and CCL2 siRNA as a positive control. The data revealed that the mRNA and protein of CCL2 were suppressed by the miR-206 mimic (Fig. 4A and B).

In conclusion, the results of the present study confirmed CCL2 as a direct target of miR-206, and showed that the upregulation of CCL2 caused by the downregulation of miR-206 was responsible for the development of severe HEV71 encephalitis.

\section{References}

1. Brown BA, Oberste MS, Alexander JP Jr, Kennett ML and Pallansch MA: Molecular epidemiology and evolution of enterovirus 71 strains isolated from 1970 to 1998. J Virol 73 9969-9975, 1999.

2. Wang YF, Chou CT, Lei HY, Liu CC, Wang SM, Yan JJ, Su IJ, Wang JR, Yeh TM, Chen SH and Yu CK: A mouse-adapted enterovirus 71 strain causes neurological disease in mice after oral infection. J Virol 78: 7916-7924, 2004.

3. Wang SM and Liu CC: Enterovirus 71: Epidemiology, pathogenesis and management. Expert Rev Anti Infect Ther 7: 735-742, 2009.

4. Wang SM, Lei HY, Yu CK, Wang JR, Su IJ and Liu CC: Acute chemokine response in the blood and cerebrospinal fluid of children with enterovirus 71-associated brainstem encephalitis. J Infect Dis 198: 1002-1006, 2008.

5. Schroeder M, Viezens L, Schaefer C, Friedrichs B, Algenstaedt $P$, Rüther W, Wiesner L and Hansen-Algenstaedt N: Chemokine profile of disc degeneration with acute or chronic pain. J Neurosurg Spine 18: 496-503, 2013.

6. Deshmane SL, Kremlev S, Amini S and Sawaya BE: Monocyte chemoattractant protein-1 (MCP-1): An overview. J Interferon Cytokine Re 29: 313-326, 2009.

7. Kikuchi T, Nakamura T, Ikeda T, Ogata $\mathrm{H}$ and Takagi K: Monocyte chemoattractant protein-1 in the intervertebral disc. A histologic experimental model. Spine (Phila Pa 1976) 23: 1091-1099, 1998

8. Yoshida M, Nakamura T, Kikuchi T, Takagi K and Matsukawa A: Expression of monocyte chemoattractant protein-1 in primary cultures of rabbit intervertebral disc cells. J Orthop Res 20 1298-1304, 2002.

9. Hammond SM: MicroRNAs as oncogenes. Curr Opin Genet Dev 16: 4-9, 2006

10. Brown BD and Naldini L: Exploiting and antagonizing microRNA regulation for therapeutic and experimental applications. Nat Rev Genet 10: 578-585, 2009.

11. Bartel DP: MicroRNAs: Target recognition and regulatory functions. Cell 136: 215-233, 2009.

12. Xiao C and Rajewsky K: MicroRNA control in the immune system: Basic principles. Cell 136: 26-36, 2009.
13. Gottwein E and Cullen BR: Viral and cellular microRNAs as determinants of viral pathogenesis and immunity. Cell Host Microbe 3: 375-387, 2008.

14. Xu LJ, Jiang T, Zhao W, Han JF, Liu J, Deng YQ, Zhu SY, Li YX, Nian QG, Zhang Y, et al: Parallel mRNA and microRNA profiling of HEV71 infected human neuroblastoma cells reveal the up-regulation of miR-1246 in association with DLG3 repression. PLoS One 9: e95272, 2014.

15. Yuan A, Li J, Liu P, Chen Z, Hou M, Wang J and Han Z: Association of interleukin- $6572 \mathrm{C} / \mathrm{G}$ gene polymorphism and serum or cerebrospinal fluid interleukin-6 level withenterovirus 71 encephalitis in Chinese Han patients with hand, foot, and mouth disease. Inflammation 38: 728-735, 2015.

16. Li JA, Chen ZB, Lv TG and Han ZL: Genetic polymorphism of CCL2-2518, CXCL10-201, IL8+781 and susceptibility to severity of Enterovirus-71 infection in a Chinese population. Inflamm Res 63: 549-556, 2014.

17. Livak KJ and Schmittgen TD: Analysis of relative gene expression data using real-time quantitative PCR and the 2(-Delta Delta C(T)) Method. Methods 25: 402-408, 2001.

18. Wong N and Wang X: miRDB: An online resource for microRNA target prediction and functional annotations. Nucleic Acids Res 43 (Database issue): D146-D152, 2015.

19. Wang X: Improving microRNA target prediction by modeling with unambiguously identified microRNA-target pairs from CLIP-ligation studies. Bioinformatics 32: 1316-1322, 2016.

20. Sharma SB, Lin CC, Farrugia MK, McLaughlin SL, Ellis EJ, Brundage KM, Salkeni MA and Ruppert JM: MicroRNAs 206 and 21 cooperate to promote RAS-extracellular signal-regulated kinase signaling by suppressing the translation of RASA 1 and SPRED1. Mol Cell Biol 34: 4143-4164, 2014.

21. Loyer X, Potteaux S, Vion AC, Guérin CL, Boulkroun S, Rautou PE, Ramkhelawon B, Esposito B, Dalloz M, Paul JL, et al: Inhibition of microRNA-92a prevents endothelial dysfunction and atherosclerosis in mice. Circ Res 114: 434-443, 2014.

22. Manoharan P, Basford JE, Pilcher-Roberts R, Neumann J, Hui DY and Lingrel JB: Reduced levels of microRNAs miR-124a and miR-150 are associated with increased proinflammatory mediator expression in Krüppel-like factor 2 (KLF2)-deficient macrophages. J Biol Chem 289: 31638-13646, 2014.

23. Nazari-Jahantigh M, Wei Y, Noels H, Akhtar S, Zhou Z, Koenen RR, Heyll K, Gremse F, Kiessling F, Grommes J, et al: MicroRNA-155 promotes atherosclerosis by repressing Bcl6 in macrophages. J Clin Invest 122: 4190-14202, 2012.

24. Bartel DP, MicroRNAs: Genomics, biogenesis, mechanism, and function. Cell 116: 281-297, 2004.

25. Baltimore D, Boldin MP, O'Connell RM, Rao DS and Taganov KD: MicroRNAs: New regulators of immune cell development and function. Nat Immunol 9: 839-845, 2008.

26. Sonkoly E, Ståhle M and Pivarcsi A: MicroRNAs and immunity: Novel players in the regulation of normal immune function and inflammation. Semin Cancer Biol 18: 131-140, 2008.

27. van Rooij E, Liu N and Olson EN: MicroRNAs flex their muscles. Trends Genet 24: 159-166, 2008.

28. Boutz PL, Chawla G, Stoilov P and Black DL: MicroRNAs regulate the expression of the alternative splicing factor $\mathrm{nPTB}$ during muscle development. Genes Dev 21: 71-84, 2007.

29. Lu C, Huang X, Zhang X, Roensch K, Cao Q, Nakayama KI, Blazar BR, Zeng Y and Zhou X: miR-221 and miR-155 regulate human dendritic cell development, apoptosis and IL-12 production through targeting of p27kip1, KPC1, and SOCS-1. Blood 117: 4293-4303, 2011

30. Zhu QY, Liu Q, Chen JX, Lan K and Ge BX: MicroRNA-101 targets MAPK phosphatase-1 to regulate the activation of MAPKs in macrophages. J Immunol 185: 7435-7442, 2010.

31. Liu Y, Chen Q, Song Y, Lai L, Wang J, Yu H, Cao X and Wang Q: MicroRNA-98 negatively regulates IL-10 production and endotoxin tolerance in macrophages after LPS stimulation. FEBS Lett 585: 1963-1968, 2011.

32. Wendlandt EB, Graff JW, Gioannini TL, McCaffrey AP and Wilson ME: The role of microRNAs miR-200b and miR-200c in TLR4 signaling and NF- $\kappa \mathrm{B}$ activation. Innate Immun 18: 846-455, 2012.

33. Banisadr G, Rostène W, Kitabgi $P$ and Parsadaniantz SM: Chemokines and brain functions. Curr Drug Targets Inflamm Allergy 4: 387-399, 2005.

34. Carr MW, Roth SJ, Luther E, Rose SS and Springer TA: Monocyte chemoattractant protein 1 acts as a T-lymphocyte chemoattractant. Proc Natl Acad Sci USA 91: 3652-3656, 1994. 
35. Hickman SE and El Khoury J: Mechanisms of mononuclear phagocyte recruitment in Alzheimer's disease. CNS Neurol Disord Drug Targets 9: 168-173, 2010.

36. Carrillo-de Sauvage MA, Gómez A, Ros CM, Ros-Bernal F, Martín ED, Perez-Vallés A, Gallego-Sanchez JM Fernández-Villalba E, Barcia C Sr, Barcia C Jr and Herrero MT: CCL2-expressing astrocytes mediate the extravasation of $\mathrm{T}$ lymphocytes in the brain. Evidence from patients with glioma and experimental models in vivo. PLoS One 7: e30762, 2012.

37. Amaral DC, Rachid MA, Vilela MC, Campos RD, Ferreira GP, Rodrigues DH, Lacerda-Queiroz N, Miranda AS, Costa VV, Campos MA, et al: Intracerebral infection with dengue-3 virus induces meningoencephalitis and behavioral changes that precede lethality in mice. J Neuroinflammation 8: 23, 2011.

38. Klein RS, Lin E, Zhang B, Luster AD, Tollett J, Samuel MA, Engle M and Diamond MS: Neuronal CXCL10 directs CD8+ T-cell recruitment and control of West Nile virus encephalitis. J Virol 79: 11457-11466, 2005

39. Savarin C, Stohlman SA, Atkinson R, Ransohoff RM and Bergmann CC: Monocytes regulate $\mathrm{T}$ cell migration through the glia limitans during acute viral encephalitis. J Virol 84: 4878-4888, 2010.
40. Phares TW, Kean RB, Mikheeva T and Hooper DC: Regional differences in blood-brain barrier permeability changes and inflammation in the apathogenic clearance of virus from the central nervous system. J Immunol 176: 7666-7675, 2006.

41. Mansfield KL, Johnson N, Nunez A, Hicks D, Jackson AC and Fooks AR: Up-regulation of chemokine gene transcripts and T-cell infiltration into the central nervous system and dorsal root ganglia are characteristics of experimental European bat lyssavirus type 2 infection of mice. J Neurovirol 14: 218-228, 2008.

42. Roy A, Phares TW, Koprowski H and Hooper DC: Failure to open the blood-brain barrier and deliver immune effectors to central nervous system tissues leads to the lethal outcome of silver-haired bat rabies virus infection. J Virol 81: 1110-1118, 2007.

43. Johnson N, Mansfield KL, Hicks D, Nunez A, Healy DM, Brookes SM, McKimmie C, Fazakerley JK and Fooks AR: Inflammatory responses in the nervous system of mice infected with a street isolate of rabies virus. Dev Biol (Basel) 131: 65-72, 2008. 